\title{
Survey on Segmentation of Brain Tumor: A Review of Literature
}

\author{
Ashima Anand ${ }^{1}$, Harpreet Kaur ${ }^{2}$ \\ Student, ECE Department, Chandigarh University, Gharuan, India ${ }^{1}$ \\ Assistant Professor, CSE Department, Chandigarh University, Gharuan, India ${ }^{2}$
}

\begin{abstract}
Segmentation of brain tumor is a vital step in the initial detection of tumor in the medical field. Although various methods have been presented for brain tumor segmentation, but enhancing tumor is a challenging task since tumor possess complex characteristics in appearance and boundaries. Brain tumor' segmentation must be done with precision in the clinical practises. The objective of this review paper presents a comprehensive overview for MRI brain tumor segmentation methods. In this paper, various techniques like region based, fuzzy clustering means (FCM), thresholding and artificial neural network (ANN) algorithms have been discussed. Comparative analysis among these various segmentation conventions has been evaluated. Finally, culmination and future aspects are addressed for brain tumor segmentation methods.
\end{abstract}

Keywords: Brain tumor, Image Segmentation, Threshold-based, Region-based, Artificial Neural Networks, FCM.

\section{INTRODUCTION}

Brain tumor segmentation is one of the competitive task to analyse the characteristics of tumor in medical treatment planning. In medical terms, brain tumor cited as Intracranial Neoplasm, is induced due to extraordinary development of brain tissues. Brain tumors are of two types:- primary brain tumors and metastatic brain tumors. The former develops in the brain and stay there only, the latter begin as a cancer elsewhere in the body and spreads to the brain. Brain tumor's mortality rate has risen and studies show that about $90 \%$ of tumors are found to be glial tumors over 20 years[1].Brain tumors vary depending upon its distinct components like location,shape, size and image intensities.

Segmentation of brain tumor takes into account the detachment of tumor tissues(tumor, edema and necrosis) from normal brain tissues: gray matter(GM), white matter(WM) and cerebrospinal fluid (CSF)[2].Brain tissues segmentation especially tumor and edema, is an intricate task because of artifacts in tumor, complex shape, heterogeneous intensity distribution and variability of the position of tumor[3].
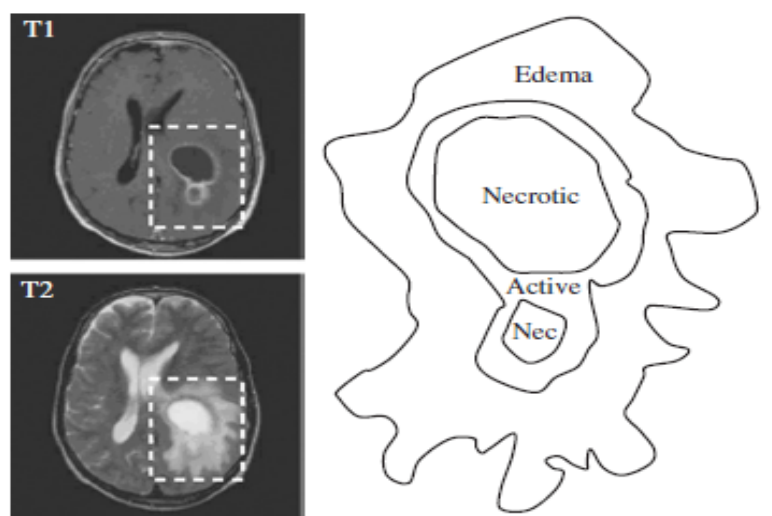

Fig. 1: Example of a brain tumor in the T1 with contrast and $\mathrm{T} 2[26]$
In recent years, medical imaging and soft computing have made significant advancements in the field of brain tumor segmentation.

This paper shows a review of various techniques and algorithms for brain tumor detection using MRI image segmentation. Semiautomatic and fully automatic methods are practical for brain tumor segmentation because errors are lower in this case as comparison to manual segmentation methods.

The outline of this paper is as follows: Section II deals with literature survey. Section III presents the summary of brain tumor segmentation methods. Section IV concludes the paper. Section V presents the future aspects.

\section{LITERATURE SURVEY}

A. Manual and Automated tumor segmentation

The classification of Brain tumor segmentation methods can be made depending on the degree of human interaction as:

A.1 Manual segmentation

It involves delineation of the boundaries of tumor manually and representing region of anatomic structures with various labels [4].Manual segmentation requires software tools for the ease of drawing regions of interest (ROI), is a tedious and exhausting task.MRI scanners produce multiple 2-D slices and the human expert has to mark tumor regions carefully, otherwise it will generate jaggy images that lead to poor segmentation results [2].

A.2 Semi-automatic segmentation

In semi-automatic brain tumor segmentation, human interaction is least as possible. According to Olabarriaga et al.[5],the semiautomatic or interactive brain tumor segmentation components consist of computational part, interactive part and the user interface. Since it involves both computer and humans'expertise, result depends on 


\section{International Journal of Advanced Research in Computer and Communication Engineering}

Vol. 5, Issue 1, January 2016

both the combination. Efficient segmentation of brain tumor is possible through this strategy but it is also subjected to variations between expert users and within same user.

\section{A.3 Fully automatic segmentation}

In this method, there is no intervention of human and segmentation of tumor is determined with the help of computer. It involves the human intelligence and is developed with soft computing techniques, which is a difficult task. Brain tumor segmentation has various properties which reduce the advantage of humans over machines. These methods are likely to be used for large batch of images in research environment. However; these methods have not gained popularity for clinical practice, due to lack of transparency and interpretability [6].

\section{B. Supervised and Unsupervised Segmentation}

Image segmentation' objective is to segregate the image into mutually exclusive regions, which are similar with respect to pre-defined subsets. This objective can be accomplished using two methods of segmentation methods- Supervised and Unsupervised methods [7].

The detailed explanations about these methods are as follows:

B.1 Unsupervised segmentation: If for training input vectors, target output is unknown, training method adopted is unsupervised learning. In the previous years, various unsupervised learning methods such as K-means and fuzzy clustering has gained popularity for brain tumor segmentation[8].The main aim of this type of segmentation is to segment the image into areas that have similar intensity and has well defined anatomic properties. Unsupervised segmentation of brain tumor achieve its anatomic goal by segmenting the image into atleast two anatomically regions, one is tumor and other is edema. The advantage of this type is that it can handle very difficult tasks such as brain tumor segmentation; it produces an accurate segmentation of different regions present in heterogeneous tumor [9]. Disadvantages of this segmentation are: number of regions is to be known before, tumors may not be specified clearly. This disadvantage can be avoided using skull stripping. Skull stripping is a pre-processing step to wipe out noncerebral tissue such as fat, muscle, skin, skull which are not desired region of interest [10].

B.2 Supervised segmentation: In supervised learning, the network is provided with series of sample inputs and output is compared with expected response. It involves both training phase that uses labelled data that maps features to labels and testing phase is used to map labels to unlabeled data[2]. The advantage of this type is that training set can be changed; it can reduce the manual task by providing labelled data. Irrespective of its advantages, it suffers from disadvantages that it requires patient specific training for brain tumor supervised segmentation and also human variability is also a concern.

\section{Segmentation methods}

In the segmentation process, accurate delineation of the tumor is responsible for early tumor diagnosis in clinical practice. Manifold approaches for brain tumor segmentation has been proposed. But no standard segmentation technique can produce satisfactory results for all imaging applications..In general, various segmentation techniques are as follows:

C. 1 Threshold-based methods

It is the convenient and basic technique of image segmentation.It convert gray scale images into binary images[11].If we consider $\mathrm{g}(\mathrm{x}, \mathrm{y})$ as the segmented image,we will get two outputs for the corresponding input image $f(x, y)$.

According to this technique,

$\mathrm{g}(\mathrm{x}, \mathrm{y})=1$; if $\mathrm{f}(\mathrm{x}, \mathrm{y})>=\mathrm{T}$

and $g(x, y)=0$;if $f(x, y)<T$, where $T$ corresponds to threshold[12].Pixels having value 1 is the desired tumor's area,whose processing is made further using morphological operations. Merit of this technique is that it differentiates between black and white intensity values.Computations required for this technique are not very complex.Drawback of this technique is that we may not get accurate results and the pixels may or may not be connected.It is classified into two types-local and global thresholding. The thresholding in which the value of $\mathrm{T}$ is constant or fixed is called global thresholding and otherwise is local thresholding.

\section{2 Region-based methods}

This method is used to examine pixels by merging neighbourhood pixels to form disjoint regions with homogeneity properties [13]. The region growing and the watershed segmentation methods are types of the regionbased methods and are mostly used in the process of brain tumor segmentation.

Region growing is the simplest region-based segmentation method and is used to extract a connected region of similar pixels from an image. It is a technique that group pixels into a larger region,by appending seeds to neighbourhood pixels[14].Initialisation of the seed point is a main aspect.It may be implemented by Mean or Max-Min algorithms. It aims to group the neighbourhood pixels in such a manner so that homogeneity is maintained.

The principle of this algorithm is as follows [14]:

1. Select the points (seed) starting in the image. These points are called germs.

2. Fix a criterion of homogeneity of the region traced for example, the grey level or intensity level.

3.Process is repeated until the desired condition is met, so that no region is grown afterwards.

\section{C.3 Artificial Neural Networks}

Artificial Neural Network (ANN) is a system modelled based on the human brain. An ANN is a network of many simple units, each having a local memory. It performs classification by learning from data and do not use any set rules. They perform well on difficult, multivariate,nonlinear and noisy domains such as brain tumor segmentation. This classifier feeds the features through a series of nodes, where mathematical operations are applied to the input nodes and a classification is done at the output 
nodes. Clarke [15] was the first researcher to propose a supervised classification using an ANN approach for brain tumor segmentation in MR images. Executing ANN for brain tumor segmentation entangle problems of complexity, the size of network becomes very large, time consuming process and large number of images are required for training the network.

A particular case of ANN is the self-organizing map (SOM). SOM is an unsupervised competitive learning algorithm.SOM automatically organizes itself according to the input data using a similarity factor like Euclidean distance [10]. The brain cortex is organized in such a manner that closer neurons will give answers to the same kind of stimulus; this is one of the reason because of which SOM technique is used in visual pattern recognition. Vijayakumar et al [16] proposed SOM method to segment tumor, necrosis, cysts, edema, and normal tissue in T2 and FLAIR MRI. Murugavalli and Rajamani presented a hybrid technique of a Hierarchical Self Organizing Map (HSOM) and Fuzzy Clustering Mean (FCM)to detect various tissues like white matter, gray matter, CSF and tumor in T1 MR images[17].

\section{C.4 Fuzzy C means (FCM)}

FCM is based on clustering which segments one class of data into two or more clusters. It works by casting each data point matching to each cluster centre on the basis of distance between the cluster and the data point. The advantages of FCM algorithm are: (1) It gives best result for overlapped data set (2) It produces comparatively better result than k-means algorithm (3) The application of FCM to MRI data has shown satisfactory results [18].

FCM is gaining popularity in the research area of brain tumor segmentation. This algorithm produces segmentation images that are clinically neuroanatomic tissue contrast from raw MRI data. A knowledge-based fuzzy clustering approach was proposed and implemented for the segmentation of the MRI images of brain tumor followed by 3-D connected components to build the tumor shape [19]. To improve the accurate detection of stage and size of tumor, a combined method of the k-means and fuzzy c-means algorithms was proposed to deal with the segmentation of brain tumor [20]. The disadvantages regarding this technique are: (1) It has a takes more computational time (2) It is more sensitive to noise.

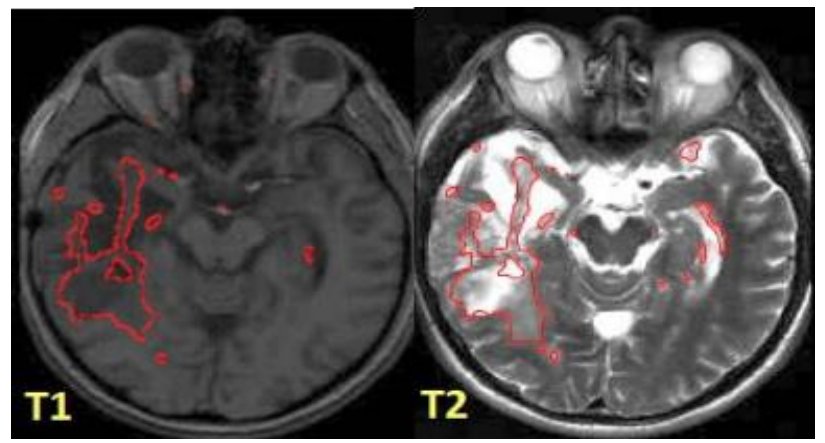

Fig 2- Examples of the results of T1 and T2 brain tumor Segmentation [27]

\section{III.SUMMARY OF BRAIN TUMOR SEGMENTATION METHODS}

\begin{tabular}{|c|c|c|}
\hline $\begin{array}{l}\text { Segmentation } \\
\text { Methods }\end{array}$ & Merits & Demerits \\
\hline $\begin{array}{l}\text { Region- } \\
\text { Based }\end{array}$ & $\begin{array}{l}\text { It is best since it } \\
\text { correctly } \\
\text { segments regions } \\
\text { that have similar } \\
\text { properties and } \\
\text { produces } \\
\text { connected } \\
\text { region[21]. }\end{array}$ & $\begin{array}{l}\text { It is quite } \\
\text { expensive in } \\
\text { terms of } \\
\text { computation of } \\
\text { both time and } \\
\text { memory. } \\
\text { Partial Volume } \\
\text { effect[22]. }\end{array}$ \\
\hline $\begin{array}{l}\text { Threshold } \\
\text { based }\end{array}$ & $\begin{array}{l}\text { Simpler,fast } \\
\text { computations and } \\
\text { lower complexity. }\end{array}$ & $\begin{array}{l}\text { Limited } \\
\text { applicability to } \\
\text { enhance tumor } \\
\text { area[23].It } \\
\text { does not take } \\
\text { into account } \\
\text { spatial } \\
\text { domain,thus } \\
\text { there } \\
\text { uncertainity } \\
\text { that regions } \\
\text { are connected. }\end{array}$ \\
\hline $\begin{array}{ll}\text { Fuzzy } & \text { C } \\
\text { Means } & \end{array}$ & $\begin{array}{l}\text { Unsupervised. } \\
\text { It converges the } \\
\text { tumor boundaries. }\end{array}$ & $\begin{array}{l}\text { Long } \\
\text { computational } \\
\text { time,sensitivity } \\
\text { to noise[24]. }\end{array}$ \\
\hline $\begin{array}{l}\text { Artificial } \\
\text { Neural } \\
\text { Networks }\end{array}$ & $\begin{array}{l}\text { Ability to model } \\
\text { non-trivial } \\
\text { distributions and } \\
\text { non-linear } \\
\text { dependence[7]. }\end{array}$ & $\begin{array}{l}\text { Gathering } \\
\text { training } \\
\text { samples is not } \\
\text { straight } \\
\text { forward and } \\
\text { learning phase } \\
\text { is slow [25]. }\end{array}$ \\
\hline
\end{tabular}

TABLE 1: SUMMARY OF BRAIN TUMOR SEGMENTATION METHODS

\section{IV.CONCLUSION}

In this paper, various segmentation techniques for the detection of brain tumor have been reviewed .Calculation of tumor's area from MRI in fast, accurate and reproducible way is a tedious task. Segmentation has proved effectively in this particular research area. Medical image processing is an active and fast-growing field. Brain tumor segmentation techniques have proved it in detecting and analysing tumors in clinical images and it will continue into the future. For distinguishing tumors from normal tissues by their image intensity, threshold-based or region growing techniques can be employed. However the accuracy on brain tumor segmentation of the proposed automated methods is quite promising, but these approaches have not gained acceptance. One of the principal reasons might be the lack of standardized procedures. Another two reasons could be the consequential differences with the traditional specialists' way of work, and the deficiency of the existing methods [2]. 


\section{International Journal of Advanced Research in Computer and Communication Engineering}

Vol. 5, Issue 1, January 2016

\section{FUTURE ASPECTS}

The future work regarding brain tumor segmentation should focus on improving the accuracy by using additional features such as prior knowledge, shape and models.To achieve better prediction rate, gradient with HPF (high pass filtering) can be used as it prominently give us edges with higher accuracy.

\section{REFERENCES}

[1] D. D. Langleben and G. M. Segall, "PET in differentiation of recurrent brain tumor from radiation injury," J. Nucl. Med., vol. 41, pp: 1861-1867, 2000

[2] Nelly Gordillo, Eduard Montseny and Pilar Sobrevilla, "State of the art survey on MRI brain tumor segmentation", Elsevier 2013, pp:113.

[3] H.-H. Chang, D. J. Valentino, G. R. Duckwiler, and A. W. Toga, "Segmentation of brain MR images using a charged fluid model," IEEE Trans. Biomed. Eng., vol. 54, no. 10, pp. 1798-1813, Oct. 2007

[4] Yao J. Image processing in tumor imaging. New techniques in oncologic imaging; 2006, pp:79-102.

[5] Olabarriage S, Smeulders A. Interaction in the segmentation of medical images:a survey.Med Image Anal 2001;5:127-42.

[6] Papageorgiou E, Spyridonos P, Glotsos D, Stylios C, Ravazoula $\mathrm{P}$, Nikiforidis G,et al. Brain tumor characterization using the soft computing technique of fuzzy cognitive maps. Appl Soft Comput 2008; 8:820-8.

[7] Schmidt M. Automatic brain tumor segmentation. Master's thesis, University of Alberta, 2005.

[8] Meiyan Huang, Wei Ynag, Yao Wu,Jun Jiang,Wufan Chen, "Brain Tumor Segemntation Based on Local Independent Projection-Base Classification",IEEE transactions on Biomedical Engineering,Vol61,No.10, October 2014,pp:2633-2645.

[9] Wiselin G, Ganesan L. Unsupervised segmentation using fuzzy logic based texture spectrum for MRI brain images. World Acad Sci Eng Technol 2005; 5:155-7.

[10] Ayse Demirhan, Mustafa Toru, and Inan Guler, "Segmentation of Tumor and Edema Along with Healthy Tissues of Brain Using Wavelets and Neural Networks, "IEEE Journal of biomedical and health informatics, vol. 19, no. 4, July 2015.

[11] Divya Kaushik,Utkarsha Singh,Paridhi Singhal and Vijai Singh, "Medical Image Segmentation using Genetic Algorithm",International Journal of Computer Applications, Volume 81,No 18,November 2013.

[12] Muhammad Ali Qadar, Yan Zhaowen, "Brain Tumor Segmentation: A Comparative Analysis", IJCSI International Journal of Computer Science Issues, Volume 11, Issue 6, No 1, November 2014, pp: 9-16.

[13] K.-P. Wong, Medical image segmentation: Methods and applications in functional imaging, in Handbook of Biomedical Image Analysis. Springer, 2005, pp. 111-182.

[14] D. Maru, B. Shah, "Image Segmentation Techniques and Genetic Algorithm", International Journal of Advanced Research in Computer Engineering and Technology, Volume 2, Issue 4, April 2013, pp: 1483-1484

[15] Clarke L. MR image segmentation using MLM and artificial neural nets. Med Phys 1991; 18(3):673

[16] Vijayakumar C, Damayanti G, Pant R, Sreedhar C. Segmentation and grading of brain tumors on apparent diffusion coefficient images using self-organizing maps. Comput Med Imaging Graph 2007; 31:473-84

[17] Murugavalli S, Rajamani V. An improved implementation of brain tumor detection using segmentation based on neuro fuzzy technique. J Comput Sci 2007;3(11):841-6.

[18] L. O. Hall, A. M. Bensaid, L. P. Clarke, R. P. Velthuizen, M. S. Silbiger, and J. C. Bezdek, A comparison of neural network and fuzzy clustering techniques in segmenting magnetic resonance images of the brain, Neural Networks,IEEE Transactions on, vol. 3, no. 5, pp. 672-682, 1992.

[19] L. M. Fletcher-Heath, L. O. Hall, D. B. Goldgof, andF. R. Murtagh, Automatic segmentation of non-enhancing brain tumors in magnetic resonance images, Artificial Intelligence in Medicine, vol. 21 , no. 1 , pp. 43-63, 2001

[20] M. P. Gupta and M. M. Shringirishi, Implementation of brain tumor segmentation in brain MR images using k-means clustering and fuzzy c-means algorithm, International Journal of Computers \& Technology, vol. 5, no. 1, pp. 54-59,2013.

[21] Salman Y, Badawi A, Assal M, Alian S. New automatic technique for tracking brain tumor response.Int Conf Biol Med Phys 2005. p. $1-4$.

[22] Sato M, Lakare S, Wan M, Kaufman A. A gradient magnitude based region growing algorithm for accurate segmentation. Int Conf Image Process 2000;3:448-51

[23] Gibbs P, Buckley D, Blackb S, Horsman MR images by morphological segmentation. Phys Med Biol A. Tumour determination from 1996 ; $41: 2437-46$.

[24] Kannan S. A new segmentation system for brain MR images based on fuzzy techniques. Appl Soft Comput 2008;8:1599-606.

[25] Iftekharuddin K, Zheng J, Islam M, Ogg R. Fractal-based brain tumor detection in multimodal MRI. Appl Math Comput 2009; 207:23-41.

[26] Corso J, Sharon E, Dube S, El-Saden S, Sinha U, Yuille A. Efficient multilevel brain tumor segmentation with integrated Bayesian model classification. IEEE TransMed Imaging 2008; 27(5):629-40 